\title{
Global University System with Globally Collaborative Environmental Peace Gaming Project
}

\author{
Takeshi Utsumi, Ph.D., P.E. \\ GLObal Systems Analysis and Simulation Association in the U.S.A. (GLOSAS/USA) \\ 43-23 Colden Street \\ Flushing, NY 11355-3998, U.S.A. \\ Tel: +1-718-939-0928 \\ utsumi@columbia.edu \\ http://www.friends-partners.org/GLOSAS/
}

\begin{abstract}
The Global University System (GUS) [1] [Utsumi, et al, 2003] is a worldwide initiative to create advanced telecommunications infrastructure for access to educational resources across national and cultural boundaries for global peace. GUS aims to create a worldwide consortium of universities to provide the underdeveloped world with access to $21^{\text {st }}$ Century education via broadband Internet technologies. The aim is to achieve "education and healthcare for all," anywhere, anytime and at any pace.
\end{abstract}

The GUS works in the major regions of the globe with partnerships of higher education and healthcare institutions. Learners in these regions will be able to take their courses from member institutions around the world to receive a GUS degree. These learners and their professors from partner institutions will also form a global forum for exchange of ideas and information and for conducting collaborative research and development with emerging global GRID computer network technology.

Globally Collaborative Environmental Peace Gaming (GCEPG) project [2] [Utsumi, 2003] with a globally distributed computer simulation system, focusing on the issue of environment and sustainable development in developing countries, is to train would-be decision-makers in crisis management, conflict resolution, and negotiation techniques basing on "facts and figures." The GUS will supply game players from around the world.

Keyword: global education, GRID, globally distributed computer simulation, environmental peace gaming

\section{BACKGROUND}

Economic interdependence among nations and cultures is spawning a global economy. Globalisation also highlights clashes of divergent cultures and belief systems, both political and religious. If global peace is ever to be achieved, global-scale education, with the use of the modern digital telecommunications, will be needed to create mutual understanding among nations, cultures, ethnic groups, and religions. The Internet is the future of telecommunications and can be a medium for building peace.

GUS has a long history of concept development and testing of multiple hardware configurations suitable for remote Internet access. These initial steps are summarized in our recent book, Global Peace Through the 
Global University System [3] [Varis, et al, 2003]. The purpose of this book is to make internationally known the philosophy, past and present actions, as well as future plans of the GUS, which have resulted from years of development and a seminal working conference at the University of Tampere, Finland, in 1999.

The editors' paper in the book, "Creating Global University System" [1] [Utsumi, et al, 2003] emphasizes the important role of higher educational institutions not only as the knowledge centers of their community but also as the gateway to the world for collaboration of creating new knowledge in global knowledge society of the $21^{\text {st }}$ Century. This paper summarizes GUS accomplishments and shows that GUS is poised to begin implementation of broadband Internet access and academic programs in remote areas of the world.

\section{GLOBAL UNIVERSITY SYSTEM}

GUS is a worldwide initiative to create satellite/wireless telecommunications infrastructure and educational programs for access to educational resources across national and cultural boundaries for global peace. GUS aims to build a higher level of humanity with mutual understanding across national and cultural boundaries for global peace. The GUS helps higher educational institutions in remote/rural areas of developing countries to deploy broadband Internet in order for them to close the digital divide and act as the knowledge center of their community for the eradication of poverty and isolation. The GUS education will promote world prosperity, justice, and peace, based on moral principles rather than political or ideological doctrines. Education and job skills are the keys in determining a nation's wealth and influence.

The GUS has task forces working in the major regions of the globe with partnerships of higher education and healthcare institutions. Learners in these regions will be able to take their courses, via advanced broadband Internet, from member institutions around the world to receive a GUS degree. These learners and their professors from participating institutions will form a global forum for exchange of ideas and information and for conducting collaborative research and development with emerging global GRID computer network technology.

\section{PROPOSED INFRASTRUCTURE}

Modern e-learning and telemedicine require high-speed access to the World Wide Web. Multi-media requirements might include two-way audio, full-motion videoconferencing up to MPEG4 quality, television-quality netcasting, and high-resolution image transfer for telemedicine. The objective of increasing quality of audio/video delivery, high interactivity, and broadband throughput can be seen as a global objective of closing the digital divide to improve e-learning and e-healthcare services in rural/remote areas of developing countries.

As diagrammed in Figure 1, GUS programs and services will be delivered via regional satellite hubs, typically located at a major university, that connect via high-speed satellite ( $45 \mathrm{Mbps})$ to educational resource cites in the E.U., U.S., and Japan. In a sense, the regional satellite hub is to be the major Internet Service Provider (ISP) for not-for-profit organizations in the region and the gateway to the outside world. The major university may also be connected to very high speed broadband Internet, as similar to the optical fiber network at $3 \mathrm{Gbps}$ of the Multimedia Broadband Internet (MBI) of the Ethiopian government, which was recently deployed with the Official Development Assistant (ODA) fund (US\$40 million) of the Japanese government <http://www.cnn.com/2005/TECH/internet/04/05/ethiopia.ap/index.html>.

Regional hubs link to branch campuses or other regional educational institutions via micro-wave ( $45 \mathrm{Mbps})$ over relatively short distances (25-50 miles). Communication from the hub and branch campuses to local sites, over distances up to 10 miles, is to be achieved by spread-spectrum wireless ( 2-10 Mbps) Internet networks, which do not require licenses in most countries.

The buildings with a broadband Internet connection will then also become relay points for the low-cost "Wi-Fi (wireless fidelity)" networks at 10 Mbps that are now rapidly appearing in Japan, USA and Europe. This 
advanced wireless communication with laptop computer will make e-learning possible for anyone, anywhere, and anytime with capabilities of Internet telephony, fax, voice mail, e-mail, Web access, videoconferencing, etc. This is not only to help local community development, but also to assure close cooperation among higher, middle and lower levels of education.

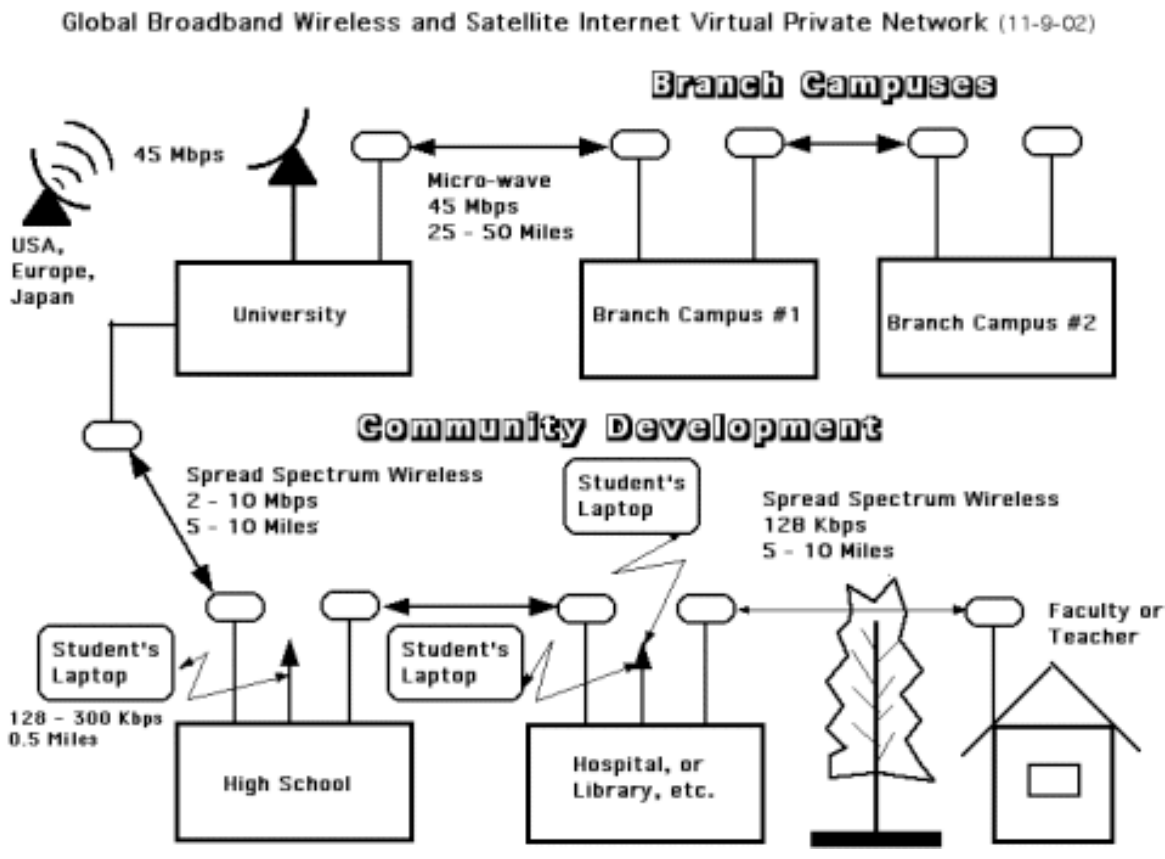

FIGURE 1

GUS is not limiting its efforts to university-level education. Some major U.S. universities are heavily involved in $\mathrm{K}-12$ education as a means to assure quality in their student pipeline. The K-12 education in many underdeveloped nations is poor to non-existent, and they produce few students who are qualified to master a university education. The seeds of poverty and terrorism are sown in children through ignorance and propaganda. What could be a more important global problem to address? Leadership must come from the universities - including, hopefully, GUS.

\section{CURRENT GUS PROJECTS}

The major university will then connect to secondary and elementary schools, libraries, hospitals, local government offices and NGOs, etc., with broadband wireless Internet at drastically discounted rates or free of charge. GUS projects are now starting in Ethiopia, Nigeria, Malawi and Ghana in Africa, Cambodia, Bangladesh and India in Asia, etc., and have received inquiries for the same from others, too.

\section{GLOBALLY COLLABORATIVE ENVIRONMENTAL PEACE GAMING (GCEPG)}

Globally Collaborative Environmental Peace Gaming (GCEPG) [2] [Utsumi, 2003] with a globally distributed computer simulation system, focusing on the issue of environment and sustainable development in developing countries, is to train would-be decision-makers in crisis management, conflict resolution, and negotiation techniques basing on "facts and figures." The Global University System will supply game players from around the world. With global GRID computer networking technology and Beowulf mini-supercomputers of cluster 
computing technology, we plan to develop a socio-economic-environmental simulation system and a climate simulation system in parallel fashion, both of which are to be interconnected in global scale - see Figure 2.

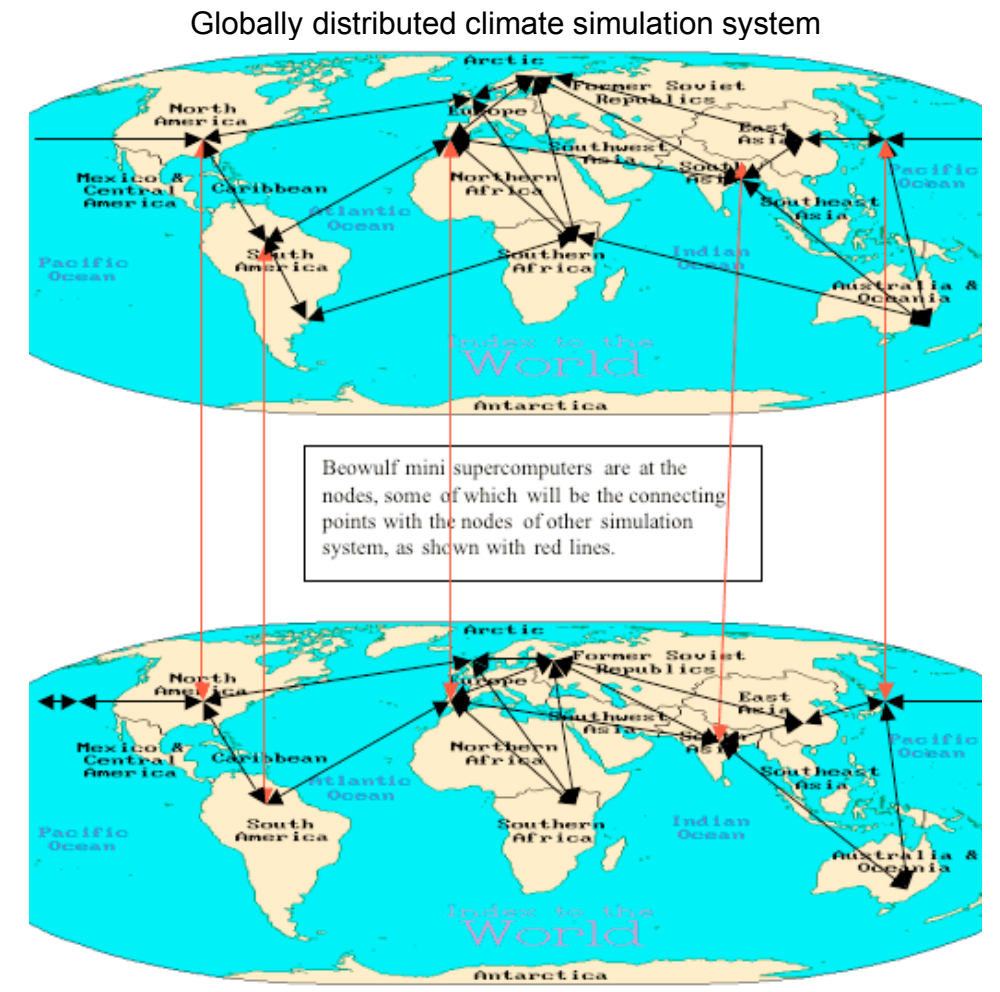

Globally distributed socio-economic-environmental simulation system

FIGURE 2: Globally collaborative environmental peace gaming networks

The GCEPG with a globally distributed computer simulation system is a computerized gaming/simulation to help decision makers construct a globally distributed decision-support system for positive sum/win-win alternatives to conflict and war. The idea involves interconnecting experts in many countries via global Internet to collaborate in the discovering of new solutions for world crises, such as the deteriorating ecology of our globe, and to explore new alternatives for a world order capable of addressing the problems and opportunities of an interdependent globe. Gaming/simulation is the best tool we have for understanding the world's interwoven problems and the solutions we propose for them. System analysis for systemic change at the global level is a precondition for any significant resolution to today's global-scale problems. The understanding gained with scientific and rational analysis and critical thinking basing on "facts and figures" would be the basis of conflict resolution for world peace, and hence ought to provide the basic principle of global education for peace.

The purpose of an interactive gaming mechanism is to help find appropriate alternative policies by establishing consensus among participating parties. It is suggested here that globally distributed computer simulation should be tested interactively with the game player inserting pseudo-policy parameters into the models whenever necessary, during the execution of simulation. This is called peace gaming/simulation [4] [Utsumi, 1977] similar to war games practiced by military strategists [5] [Schram et al., 1971]. With the advent of global broadband Internet and standard interface protocols for interconnecting various dispersed, dissimilar host computers, the potential exists for ensuring the coordination of international efforts by providing more frequent communications and an environment for shared development, enabling more credible simulation study than was previously possible. 
The GCEPG project proposes to utilize the semantic benefits of gaming simulation on a global scale to aid decision makers in appreciating the impact of their decisions on interwoven global problems, i.e., the construction of Globally Distributed Decision Support System (GDDSS) with Distributed Computer Simulation Systems (DCSS), which deals with coordination of the distributed sub-models and their experts via the global Internet for global crisis and ecology management for plus sum, peace game. Senator Fulbright once said;

"Learning together and working together are the first steps towards global peace."

\section{COMPUTER SIMULATION MODELS}

Since I created Summer Computer Simulation Conference (SCSC) in Denver, CO in 1970, myriad of simulation models in almost every facets of our globe appeared. There are three major methodologies of socio-economic modeling; (1) econometric modeling (initiated by Professor Lawrence R. Klein of University of Pennsylvania and an economic Nobel Laureate), (2) input-output modeling (initiated by Professor Wassily Leontief of New York University, an economic Nobel Laureate and a panelist of our Nagoya/Japan "Global Lecture Hall (GLH) ${ }^{\mathrm{TM}}$, videoconferencing on environmental issues from New York), and (3) system dynamics modeling (initiated by Professor Jay W. Forrester of M.I.T.).

Prof. Onishi [6] [Onishi, 2003] has already indicated his strong willingness to cooperate with this GCEPG project as providing his Futures of Global Interdependence (FUGI) model, the world largest econometric model. When we conducted US/Japan foreign trade peace gaming at the conference on "Crisis Management and Conflict Resolution" in New York City in July of 1986, we used it as a single simulation model residing in a supercomputer in Tokyo and we asked him to execute his model with the alternative policy parameters according to the progress of our gaming scenario proposed by noted U.S. economists (Prof. Lester C. Thurow of M.I.T., Provost William Nordhaus of Yale University, Mr. Keith Johnson of Townsend and Greenspan Company).

However, this time, his FUGl's sub-models will be split and be dispersed to the countries where the sub-models belong. We will arrange GUS in various countries to host the sub-models of their countries - along with construction and maintenance of its databases, revision and modification of their sub-models, and supply of game players in cooperation with their overseas counterparts through the global neural (or GRID) computer network.

Prof. Forrester has also indicated to me that his System Dynamics Group already constructed a US national model, which may be used in conjunction with FUGI model. The Millennium Institute $<$ http://www.millenniuminstitute.net/> in Arlington, VA also has national system dynamics models of Bangladesh, China, Ghana, Guyana, Italy, Malawi, Somaliland, Tunisia, and the United States. As soon as we establish our GUS in these countries, we may ask their cooperation to tie together those national models. Dr. G. O. Barney, the founder of the institute, once presented their work from his office during our GLH videoconference held in Florianopolis, Brazil in the summer of 1996.

Incidentally, after contributing to the early development of digital computers and inventing magnetic-core memory, Prof. Forrester pioneered "system dynamics," a computer simulation methodology for understanding complexity that extends far beyond servomechanisms and Cybernetics theory. He applied quantitative, system analysis and computer simulation technology to complex socio-economic, bio- and eco-systems to evaluate how alternative policies affect growth, stability, fluctuation, and changing behavior.

The system dynamics' cause-and-effect analysis based on feedback theory, along with computer simulation modeling, is the best tool to understand the inter-relatedness and inter-dependency of various complex world phenomena.

Under Forrester's leadership, pioneering schools are creating a new kind of pre-college education, starting in kindergarten that is built on a system dynamics foundation. Such education becomes inter-disciplinary with the same computer simulation concepts applied to the environment, biology, history, literature, and economics. We 
can expect future leaders with expanded abilities for crisis management, policy-making, and negotiation skills for corporate, national, and global issues. The resulting deeper understanding of social and economic complexity, arising from this new kind of education, will enhance mutual understanding among people of different countries and cultures, and facilitate world peace and a sustainable development of humankind in the 21 st century.

As millenniums of human history tell, those people in Central America, Peru, Egypt, India, China, etc., who could predict future with the celestial movements, assisted the decision-makings of kings and rulers. Future leaders of global village need to have the capability of understanding the computer simulation of socio-economicenvironmental system, since it will assist them (hopefully, even at local level) with rational analysis and critical thinking basing on "facts and figures."

\section{GRID TECHNOLOGY}

Many now consider GRID technology as the next generation Internet, which concept I initiated in 1972 [7] [McLeod, 2000]. It has demonstrated all of the effectiveness in the scientific domains as becoming a de-facto eScience technology infrastructure. This technology promises to do what the Internet has done with data on the applications. GRID computing extends the scope of distributed computing to encompass large-scale resource sharing, including massive data-storages, high-performance networking and powerful computers, highly expansive equipments (i.e., microscopes, telescopes, 3D Cave), etc. GRID technology defines a new powerful computing paradigm by analogy to the electric Power Grid. Users of the GRID will then be able (a) to use his/her private workplace to invoke any application from a remote system, (b) to use the best suited system for executing their desired particular application, (c) to access data securely and consistently from remote sites, (d) to exploit multiple systems to complete complex tasks in an economical manner, or (e) to use multiple systems to solve large problems that exceed the capacity of a single one. In this vision, the sharing doesn't mean simply exchange of data or files but rather a concrete access to resources (e.g., computers, software, data, etc.).

E-mail and multimedia World Wide Web of Internet so far contributed significantly to the world society on the dissemination of information. The next phase of the Internet development with global neural (or GRID) computer networks should be the globally collaborative experiential (the so-called "hands-on") learning and constructive creation of wisdom with interactive actions on virtual reality simulation models of joint global research and development projects on various subjects. It is said "knowledge applied with interaction becomes wisdom." The principle of the $21^{\text {st }}$ century education should be inheriting wisdom more than the mere transfer of knowledge.

Here, in 1981, I coined the phrase "Global Neural Computer Network" in which each participating game player, with his/her own desktop computer, database and sub-model, would correspond to a neuron, router to synapses, with the Internet serving as nerves in a global brain. Vice President Al Gore used this term in a speech (as the result of one of his staffs at the White House received numerous e-mail messages from my listserve) and continued with the following words:

"The Department of Defense is investing well over $\$ 1$ billion in the development and implementation of networked distributed interactive simulation. This technology, which allows dispersed learners to engage in collaborative problem solving activities in real time, is now ready for transfer to schools and workplaces outside of the defense sector." (Speaking to communications industry leaders, January 11, 1994, Washington, D.C.)

He then proposed the Digital Earth Program to assist the Mission to Planet Earth of the NASA, as one of his proposed Global Marshall Plan. This is to organize a worldwide education program to promote a more complete understanding of the global environmental crisis with a similar "massively parallel" distributed computer simulation system as our GCEPG project [8] [Gore, 1992]. He said;

"Most experts in the United States and Japan now believe in the inherent advantages of a computer architecture or system design known as massive parallelism, and massively parallel computers will undoubtedly play a key role in Mission to Planet Earth." 


\begin{abstract}
"[T]hat I am recommending here is to distribute the information collecting and processing capability in a 'massively parallel' way throughout the world by involving students and teachers in every nation. This way, some of the essential work may well be accomplished much faster and much more efficiently - and we can then work to upgrade and improve the information handling capacity in each location. Furthermore, we ought to be establishing environmental training centers and technology assessment centers throughout those areas of the world (especially the Third World) where major environmental remediation efforts are needed and where major technology transfer from the West are expected."
\end{abstract}

\title{
8. ASYNCHRONOUS GRID FOR GLOBAL PEACE GAMING
}

It is now possible to combine existing technologies to make sophisticated and more holistic explorations of various scenarios for solving global social problems. Many small computers in different countries can be interconnected, through globally distributed network and information processing, into modeling and simulation instruments for playing peace games on the scale of Pentagon war games [9] [McLeod, 1987].

About two decades ago, I proposed the development of global decision support system with globally distributed interactive gaming simulation for global socio-energy-economic system with the use of global data telecommunication network (e.g., Internet nowadays). Interconnection of dissimilar computers and models for peace gaming on energy, resources and environmental (ERE) systems, architectures for linking heterogeneous computers were outlined. The reference also described communication procedures through multi-party gaming simulation [10] [Utsumi and DeVita, 1982].

I then examined the application of the new development in the area of distributed systems and Computer Aided Communication (CAC) to the analysis of the global sociological and economical issues. Based on the review of the past attempts and experiences with model acceptance and validation, meaningful and credible simulation has to be implemented as a modeling network composed of a large number of locally developed and verified models. No single model, developed by a local group of experts has a chance for universal acceptance when it deals with controversial and confrontation-prone area such as global resource allocation and economical policies. A typical example was the case of The Limit to the Growth of the Club of Rome [11] [Meadows, 1972], though it pioneered and widely spread the usefulness of computer simulation applied to social and global affaires and phenomena.

Yet, a comprehensive model of global resources, ecology, and economy is needed for the rational management of ecosystems and for economic cooperation between nations and economic blocks. As a solution to the dilemma between the need for a unified model and a diversity of views and the special interests of diverse groups, a public Open Modeling Network (OMN) was proposed which would consist of models developed by local experts interconnected by global Internet [12] [Utsumi, et al., 1986].

The problem of managing the variety of heterogeneous models, each operating locally, yet affected from time to time by the results of similar runs at other locations, was compared to Scheduling Algorithm problem which is required by all asynchronous distributed systems consisting of the distributed communicating processors, in particular the application of Time Warp algorithm [13] [Jefferson, 1984] and the Virtual Time concept that allows organization of the information exchange among dispersed, dissimilar computational resources with asynchronous and parallel executions. The rollback mechanism for asynchronous scheduling of the Time Warp algorithm is particularly necessary for the peace gaming in global scale, because there is unavoidable conditions as (a) Time difference among game players due to the roundness of globe, (b) Latency of signal of distributed simulation models to/from geo-synchronous satellite, (c) Head-scratching time of game players for democratic decision-making with consensus.

\section{GLOBALLY COLLABORATIVE EXPERIENTIAL LEARNING WITH ELEGI AND NOMADIC}

European Learning GRID Infrastructure (ELeGI) Project [14] [Allison, et al, 2003], which is now funded by the European Commission, aims to design and implement advanced service-oriented GRID-based software 
architecture for learning. This project will develop a new paradigm focused on knowledge construction using experiential based and collaborative learning approaches in a contextualized, personalized and ubiquitous way. This will replace the current information transfer paradigm, which is based on content, and on the key authoritative figure of the teacher who provides information.

GCEPG project could be a complete and powerful demonstrator of ELeGI Project to show (1) the advantages coming from using advanced technologies (i.e., GRID for accessing to computing resources and collaboration environments) for supporting simulations execution, data analysis, etc., and (2) simulations for learning through the definition of innovative pedagogical models (i.e., socio-constructivist contextualized learning approach), and (3) to show all the benefits coming from the harmonized and synergistic use of advanced technologies together with innovative pedagogical models for learning (i.e., ELeGI).

Another project "Knowledge Management over a Digital Communication Space (Proposal acronym: NOMADIC)" is the outgrowth of the ELeGI and is based at the University of Rome. This is for the consortium of 9 prominent European organizations including the University of Tampere, Finland, and will explore the frontier of applying the most advanced web and GRID networking technologies to e-learning and e-healthcare/telemedicine. Within this NOMADIC project, our GUS and GCEPG projects will be administered at the University of Tampere, Finland.

The cooperation with those ELeGI and NOMADIC projects will assure the development of globally collaborative experiential, distributed learning with globally distributed simulation system for joint research and development on various subjects by youngsters around the world. This will then foster their creativity, and hence promoting mutual understanding among them, also, -- which is the first step toward the global peace.

\section{EXPECTED BENEFITS}

With rapid advancement of computer simulation with GRID computing network technology, such a network of mini-supercomputers around the world can also be used by researchers, even in developing countries to perform with their counterparts in developed countries for joint collaborative researches with virtual reality and virtual laboratory of various academic and engineering subjects. They can also be used in micro-biology, meteorology, chemical molecular study, DNA analysis, medicine/bioscience, 3D animation of human anatomy, agriculture, commerce, finance, nanotechnology, joint advanced engineering design, astronomy, etc. [15] [Sterling, 2001].

In a sense, our GUS/UNESCO/UNITWIN Networking Chair project aims to construct global scale knowledge forum with advanced Information and Communication Technology (ICT), i.e., with the use of massive parallel processors of globally distributed and yet interconnected mini-supercomputers through global neural computer network. This will be a paradigm shift of research and development in global scale, out of the so-called "Ivory Tower" approach.

It is expected that GUS will provide the following benefits to students and participating universities:

- Broadband Internet connection, supporting modern distance education via the World Wide Web

- Help member universities build a network of facilitators to support e-learners

- Learners may take courses from different member universities, obtaining their degree from the GUS, thus freeing them from being confined to one academic culture of a single university or country

- Learners and faculties can promote the exchange of ideas, information, knowledge, and joint research and development of Web-based teaching materials

- Researchers in developing countries can partner with colleagues in more advanced countries, and perform joint collaborative research and development with the use of virtual reality/virtual laboratories for experiential/constructive learning and creation of knowledge through the emerging global GRID computer networking technology

- Learners, faculties, and public policy makers can promote community development and many other advances at a local, regional and even on a global scale. 


\section{ORGANIZATION}

GUS is headquartered at the Global E-learning Center at the University of Tampere in Finland, under the direction of the UNESCO/UNITWIN Networking Chair, held by Dr. Tapio Varis. Currently, institutions with faculty members who are participating in GUS development projects include the University of Tampere, UK Open University, 6 federal universities of Amazonia, Havana Institute of Technology, University of Malawi, Uganda National Council for Science and Technology, McGill University in Canada, University of Tennessee in Knoxville, Cornell University, Texas A\&M University, Maui Community College, University of Milan, University of Salerno, University of Twente, Catalunyan Open University, and many others in Ethiopia, Nigeria, etc. GUS will serve as an educational broker for universities, thus helping them gain international influence and access to students that they would otherwise not reach. Those institutions affiliated with GUS become members of the GUS/UNESCO/UNITWIN Networking Chair Program.

\section{FINANCING GUS}

During the Okinawa Summit in July 2000 , the Japanese government pledged US $\$ 15$ billion to close the digital divide in developing countries and for the eradication of poverty and isolation. During the G8 Summit in Canada in June of 2002, and at the Environment Summit in South Africa in September of 2002 they also pledged US\$2 billion to aid education and healthcare in developing countries, respectively.

GUS projects will combine (1) the Japanese government's Official Development Assistance (ODA) funds and (2) Japanese electronic equipment with (a) the Internet technology and (b) content development of North America and Europe.

\section{CONCLUSIONS}

The GUS program is a comprehensive and holistic approach to building smart communities [16] [Eger, 2003] in developing countries for e-learning and e-healthcare/telemedicine. Initiatives are underway to create the necessary infrastructure and educational liaisons, and some near-term educational access is expected.

GUS and GCEPG are clearly ambitious programs, one that cannot be achieved by any one group, university, or national government. The programs require substantial collaborative contribution of ideas, expertise, technology resources, and funds from multiple sources. Those who value the visions of GUS and GCEPG are invited to join this great and noble enterprise.

REFERENCES (All URL below were retrieved on April 6, 2005)

[1] Utsumi, T., Varis, T., and Klemm, W. R., (2003), Creating Global University System, Global Peace Through The Global University System, University of Tampere Press, Tampere, Finland http://makeashorterlink.com/?I2F231019

[2] Utsumi, T., (2003), Globally Collaborative Environmental Peace Gaming, Global Peace Through The Global University System, University of Tampere Press, Tampere, Finland http://makeashorterlink.com/?E1D121E09

[3] Varis, T., Utsumi, T., and Klemm, W. R., (Editors), (2003), Global Peace Through The Global University System, University of Tampere Press, Tampere, Finland, November, (ISBN 951-44-5695-5) http://makeashorterlink.com/?M2D252E09

[4] Utsumi, T., (1977), "Peace game," Simulation, November, pp. 135

[5] Schram, S., Marks, H., Behrens, W., Levin, G., and McLeod, J., et al., (1971), Macro-system simulation, Panel Discussion Session at the 1971 Summer Computer Simulation Conference (SCSC), 1972 SCSC Proceedings, Society for Computer Simulation, pp. 1491-1502 
[6] Onishi, A., (2003), UNESCO EOLSS (Encyclopedia of Life Support System) Theme 1.47: Integrated Global Models for Sustainable Development, Oxford, U.K., EOLSS Publisher

[7] McLeod, J., (2000), "Power (?) Grid!," Simulation in the Service of Society, Simulation, September http://makeashorterlink.com/?H241159B9

[8] Gore, A., (1992), Earth in the Balance, Houghton Mifflin Company, New York, pp. 358-360

[9] McLeod, J., (1987), "TAK is TICKING," Simulation, December, pp. 273-4

[10] Utsumi, T. and DeVita, J., (1982), GLOSAS Project (GLObal Systems Analysis and Simulation), In S. Schoemaker (Editor), Computer Networks and Simulation II, North-Holland Publishing Company, Amsterdam, pp. 279-326

[11] Meadows, Donella H., et al, (1972), The Limits to the Growth, Universe Books, New York

[12] Utsumi, T., Mikes, P. O., and Rossman, P., (1986), Peace Games with Open Modeling Network, In S. Schoemaker (Editor), Computer Network and Simulation III, Elsevier Science Publisher B.V., (NorthHolland), Amsterdam, pp. 267-298

http://makeashorterlink.com/?E59012A5A

[13] Jefferson, D. and Motro, A., (1984), The Time Warp Mechanism for Database Concurrency Control, Technical Report TR-84-302, University of Southern California, Los Angeles, January

[14] Allison, C., et al, (2003), Human Learning as a Global Challenge: European Learning GRID Infrastructure, Global Peace Through The Global University System, University of Tampere Press, Tampere, Finland http://makeashorterlink.com/?Z1D031A39

[15] Sterling, T., (2001), "How to Build a Hyper Computer," Scientific American, July, Pages 38-45

[16] Eger, J., (2003), Athens in the Information Age, Global Peace Through The Global University System, University of Tampere Press, Tampere, Finland http://makeashorterlink.com/?N38D25259

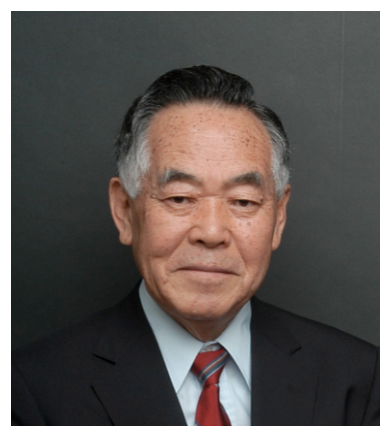

Dr Takeshi Utsumi is the Founder and Vice President for Technology \& Coordination of Global University System (GUS) and the Chairman of the GLObal Systems Analysis and Simulation Association in the U.S.A. (GLOSAS/USA). He is the 1994 Laureate of the Lord Perry Award for Excellence in Distance Education. His public services have included political work for deregulation of global telecommunications and the use of e-mail and voice over Internet Protocol (VolP) through ARPANET, Telenet and Internet; helping extend American university courses to developing countries; the conduct of innovative distance teaching trials with "Global Lecture Hall $(\mathrm{GLH})^{\mathrm{TM}}$ " multipoint-to-multipoint multimedia interactive videoconferences using hybrid technologies; as well as lectures, consultation, and research in process control, management science, systems science and engineering at the University of Michigan, the University of Pennsylvania, M.I.T. and many other universities, governmental agencies, and large firms in Japan and other countries. 